\title{
The diagnostic performance of non-contrast T1- mapping in patients with acute myocarditis on cardiovascular magnetic resonance imaging
}

\author{
Vanessa Ferreira ${ }^{12^{*}}$, Stefan K Piechnik', Erica Dall'Armellina' ${ }^{1}$,Theodoros Karamitsos ${ }^{1}$, Jane M Francis ${ }^{1}$, \\ Robin P Choudhury ${ }^{1}$, Attila Kardos ${ }^{3}$, Matthias G Friedrich ${ }^{4,2}$, Matthew D Robson', Stefan Neubauer ${ }^{1}$ \\ From 15th Annual SCMR Scientific Sessions \\ Orlando, FL, USA. 2-5 February 2012
}

\section{Summary}

Non-contrast T1-mapping using ShMOLLI can serve as a novel CMR diagnostic criterion in patients presenting with suspected acute myocarditis.

\section{Background}

The accurate diagnosis of acute myocarditis on cardiovascular magnetic resonance imaging (CMR) often requires multiple modalities, including $\mathrm{T} 2$-weighted $(\mathrm{T} 2 \mathrm{w})$, early and late gadolinium imaging. T1-mapping is an emerging technique which is also sensitive to acute changes in free water content. We hypothesized that non-contrast T1-mapping using the novel Shortened Modified Look-Locker Inversion Recovery (ShMOLLI) sequence can serve as a new diagnostic criterion for acute myocarditis.

\section{Methods}

We studied 23 patients with suspected acute myocarditis and 17 healthy controls. All patients presented with chest pain and troponin I $>0.04 \mathrm{ug} / \mathrm{L}$. CMR within 7 days included (1) T2w short-TI inversion recovery (STIR);(2) ShMOLLI T1-mapping; and (3) phase-sensitive late gadolinium enhancement (LGE) (Fig 1). T2 signal intensity (SI) relative to skeletal muscle (T2 SI ratio) and absolute $\mathrm{T} 1$ values per-subject were analyzed.

\section{Results}

All patients had a CMR diagnosis of acute myocarditis based on both positive T2-STIR and typical LGE pattern. Compared to controls, both mean T1 and T2 SI ratio in patients were significantly higher $(\mathrm{T} 1=1036$ $\pm 71 \mathrm{~ms}$ vs. $\mathrm{T} 1=938 \pm 19$; $\mathrm{T} 2$ SI ratio $=1.77 \pm 0.24$ vs. 1.52 \pm 0.10 , $\mathrm{p}<0.0002$ for both). Receiver operator characteristics analysis showed excellent diagnostic performance for both methods: the area-under-the-curve for ShMOLLI T1-mapping $=0.96$ and STIR $=0.93(p=0.3$, Fig 2 ). The equal sensitivity and specificity points were $\mathrm{T} 1=87 \%(\mathrm{~T} 1=958 \mathrm{~ms})$ and $\mathrm{T} 2=83 \%$ (T2 SI ratio $=1.63)$.

\section{Conclusions}

Non-contrast T1-mapping using ShMOLLI has a high diagnostic performance for acute myocarditis and may be used as a novel additional CMR diagnostic criterion.

\section{Funding}

This study is funded by the Oxford National Institute for Health Research Biomedical Research Centre Programme. VMF is funded by the Alberta Heritage Foundation for Medical Research (AHFMR) and the University of Oxford Clarendon Fund Scholarship. Dr. Robin Choudhury is a Wellcome Trust Senior Research Fellow in Clinical Science. Stefan Neubauer and Robin Choudhury acknowledge support from the British Heart Foundation Centre of Research Excellence, Oxford.

\footnotetext{
Author details

${ }^{1}$ Cardiovascular Medicine, University of Oxford, Oxford, UK. ${ }^{2}$ Stephenson Cardiovascular MR Centre, Libin Cardiovascular Institute of Alberta, University of Calgary, Calgary, AB, Canada. ${ }^{3}$ Cardiology, Milton Keynes NHS Hospital Foundation Trust, Milton Keynes, UK. ${ }^{4}$ Cardiology, Université de Montréal, Montréal, QC, Canada.
}

Published: 1 February 2012

${ }^{1}$ Cardiovascular Medicine, University of Oxford, Oxford, UK

Full list of author information is available at the end of the article

(C) 2012 Ferreira et al; licensee BioMed Central Ltd. This is an open access article distributed under the terms of the Creative Commons 


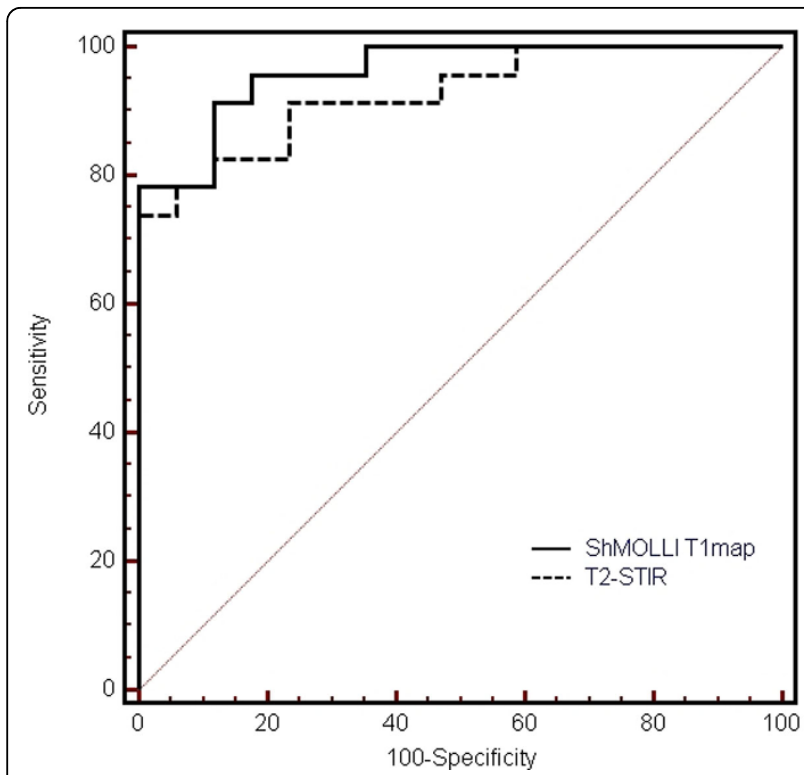

Figure 1 ROC curves for ShMOLLI T1-mapping and T2-STIR in acute myocarditis.

doi:10.1186/1532-429X-14-S1-P179

Cite this article as: Ferreira et al.: The diagnostic performance of noncontrast T1-mapping in patients with acute myocarditis on

cardiovascular magnetic resonance imaging. Journal of Cardiovascular Magnetic Resonance 2012 14(Suppl 1):P179.

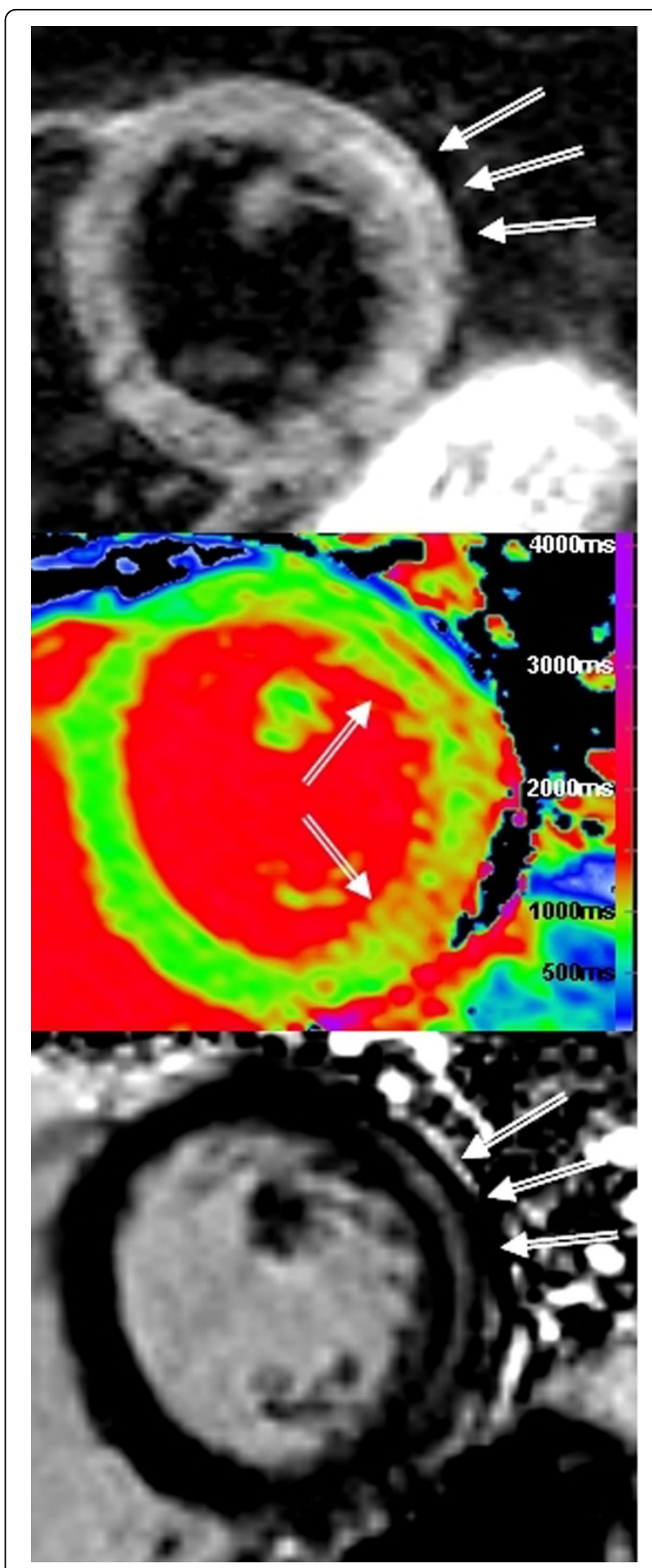

Figure 2 Acute myocarditis. (Top) STIR demonstrating increased signal intensity in the mid lateral wall. (Middle) ShMOLLI T1-map demonstrating increased T1 values (1100-1200 ms) in the lateral wall. (Bottom) LGE imaging demonstrating mid-wall enhancement in the lateral wall. 